\title{
The Growth and Behavior of a Virtual Life by using Genetic Algorithm
}

\author{
Min-Su Kwon*, Do-Wan Kim**, Hoon Kang*** \\ School of Electrical and Electronic Engineering, Chung-Ang University
}

\begin{abstract}
In this paper, we modeled a virtual life (VL) that reacts to the user's action according to its own behavioral characteristics and grows itself. We established some conditions with which such a VL is designed. Genetic Algorithm is used for the growth process that changes the VL's properties. In this process, the parameter values of the VL's properties are encoded as one chromosome, and the GA operations change this chromosome. The VL's reaction to the user's action is determined by these properties as well as the general expectation of each reaction. These properties are evaluated through 5 fitness measures so as to deal with multi-objective criteria. Here, we present the simulation of the growth process, and show some experimental results.
\end{abstract}

Key Words : Virtual Life, Genetic Algorithm, Growth Process, Artificial Life, Behaviors and Reaction

\section{Introduction}

In these days, due to the vast internet infra and the powerful computing speed, we easily experience the online virtual environments, such as online games, avatar chatting and 3D shopping malls from which many peoples and companies induce some value-added profits.

The aims of our study are to make the virtual life (VL) that sympathizes of human and behaves itself like a real creature in the virtual environment. In this paper, we design a virtual life that react to user's action according to it's own properties and grows itself as an artificial life form. We choose a puppy-like artificial creature, a most intimate friend with the human, for a candidate of a VL. A user grows one's own VL in the virtual environment. We define some conditions, with which such a VL must possess, and it is simulated according to those conditions.

First, the VL should act differently according to its own properties. Second, the VL's properties are to be changed interactively with a user. Such evolving properties grow the VL. Finally, the VL's properties, converging to one direction, must not be changed easily as a user provides other action for it and leads the properties into another direction.

Among computational techniques of artificial intelligence, we use Genetic Algorithm (GA) [1],[2] for the growth process of the Virtual Life due to its simplicity and effectiveness.

GA was proposed by John Holland in early 1970s. It is a search algorithm based on the mechanics of natural selection and evolving genetics. GA has been found to be

접수일자 : 2003년 1월 27일

완료일자 : 2003년 9월 22일

This work was supported by grant no. R01-2000-00000277-0 from the Basic Research Program of the Korean Science \& Engineering Foundation (KOSEF). one of the most flexible, efficient and robust among all search algorithms known to artificial intelligence. Because of these properties, the method is now widely used to solve a broad range of different optimization problems.

Interactive Genetic Algorithm (IGA)[3] is an optimization method that adopts GA among system optimization based on the subjective human evaluation. It is simple GA technique that fitness function is interactively replaced or evaluated by a user. In most IGA systems, the fitness value is assigned to each individual in the population by the human subjective evaluation instead of using the chosen fitness function, Then, GA optimizes the target system to obtain the preferred output based on the user's evaluation.

\section{The Growth Process of VL}

In this section, we will explain the growth process of the VL using GA. The VL grows under the influence of user's action after it has been created. The VL's growth is composed of variation of two special properties, the personality and the body.

\subsection{Chromosomes and Population}

The VL's properties are composed of two behavioral properties and three physical properties. These properties are coded as the chromosomes, its parameters are shown in Table 1.

Table 1. Chromosomes of Virtual Life

\begin{tabular}{|c|c|c|c|c|}
\hline \multicolumn{3}{|c|}{ Phenotype (Properties) } & \multicolumn{2}{c|}{ Genotype } \\
\hline \multirow{2}{*}{ Tendency } & Docility & $-63 \sim+64$ & 7bit & \\
\cline { 2 - 5 } & Activity & $-63 \sim+64$ & 7bit & \multirow{3}{3}{32 bit } \\
\hline \multirow{3}{*}{ Body } & Fatness & $-63 \sim+64$ & 7bit & binary \\
\cline { 2 - 4 } & Physical Strength & $-63 \sim+64$ & 7bit & string \\
\cline { 2 - 4 } & Stature & $-7 \sim+8$ & 4 bit & \\
\hline
\end{tabular}


Each property acts as the following roles. The docility is the most important property. It determines the VL's behavior. If this value is high, the VL behaves obediently. If this value is low, it shows a violent action.

The activity is the parameter of positiveness in reaction. The VL behaves briskly if its value is greater or equal to 0 while it shows a negative action if its value is lower than 0 .

The fatness is relatively less effective than above 2 behavioral parameters but it has an effect on the external form as the VL becomes embodiment in graphics. When the value is 0 , it has a general form; if the value is greater than 0 , it is in a fat form; and if the value is low than 0 , it is in a skinny form. Its effect that gives in action is a counterwork against the activeness but the effect is less than that of the activeness.

The physical strength displays the condition of the VL's health. It is relatively less effective than the 2 behavioral parameters. However, if the physical strength is lower than a regular value, the VL becomes dead in the end. Therefore, it is one of the most important properties.

Finally, the stature is a parameter, which has no influence on VL's action, but is used graphically. The VL grows up as time passes with a change of the stature parameter. If the value is higher than 0 , the $\mathrm{VL}$ is longer than a standard stature, and if the value is lower than 0 , it becomes shorter.

These five properties represent the VL's status that changes according to the user's action. The Docility, the activeness, the Fatness and the physical strength determine the VL's reaction to the user's action. On the other hand, the stature is used to express the external feature form.

Therefore, the genotypes of the four properties are encoded by 7 bit binary numbers between -64 and +64 . Otherwise, the genotype of the stature is represented by 4 bit binary code between -8 and +8 .

Therefore, the VL's properties are stored in a variable of 4 bytes ( 32 bits) in this composition. With GA, a VL has a total 100 chromosomes. Initially, 100 chromosomes are generated by the uniform random numbers. Therefore, a VL born at the first time shows a very unstable reaction and it converges to a direction that corresponds with the user's action.

\subsection{Fitness Evaluation Based on User's Action}

Each chromosome gets the fitness value from the user's action, and the effect that user's action gives in VL's properties is shown in Table 2.

These numerical values are determined by experiments. Embracing, stroking, neglecting, shouting, threatening and hitting in Table 2 let the population evolve to the next generation each time these happen. Feeding and exercising give an effect to the VL at the end of a day. The number of times of these actions in a day is counted, and then the operations are performed. The fitness of each chromosome is calculated by the next formula.

$$
\begin{aligned}
& F_{i}=\left(f_{i}-f_{\min }\right)+\frac{F_{\max }-F_{\min }}{K-1} \\
& f_{i}=\sum_{\text {all } j}\left(V_{j} \times T_{j}\right)
\end{aligned}
$$

$f_{i}$ : the fitness value of $i$ th chromosome

$f_{\min }$ : the minimum fitness value among

$f_{\min }$ chromosomes

$f_{\max }:$ the maximum fitness value among

population

$F_{i}$ : the last fitness value of $i$ th chromosome

$K$ : the selection pressure

$V_{j}: j$ th property value of chromosome $T_{j}: \begin{aligned} & \text { the proportional factor of Table } 2 \text { 's } j \text { th } \\ & \text { property }\end{aligned}$

Table 2. The effect that user's action gives in VL's properties

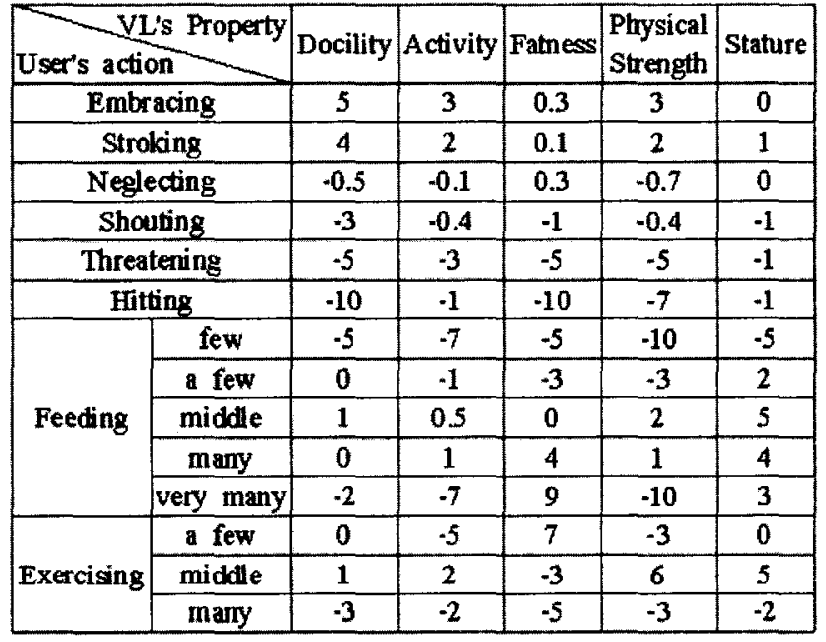

The greatest fitness value of the whole individuals becomes $\mathrm{K}$ times of the smallest fitness value in this formula, and we can keep the balance between the variance of the population and the convergence speed by controlling the selection pressure $\mathrm{K}$. In the case of feeding, when the user do not give food continually, it causes a fatal effect, but the feeding is shown to be less effective than other actions because it runs the operations just once for the specified period of time. When the user does not give food for the certain period, we can regulate the reflection ratio by lowering the selection pressure of other actions and by increasing the selection pressure of the feeding, in order to solve the problem.

\subsection{Crossovers and Mutations}

The Simple GA operations such as crossovers and mutations are used here. We select the chromosomes of 50 pairs through the roulette selection[4] according to the fitness value calculated in Section 2.2 , and these chromosomes create a 100 offspring generation to replace 
the parent generation through the crossovers and mutations.

The fitness value changes each time as the user's action differs. Therefore, we do not use the method that some parent generation's individuals are passed to the offspring generation like the elite conservation. The number of the crossover sites is set between 1 and 5 at random on each pairs. The mutation is performed on a bit-by-bit basis with probability and the mutation rate is $0.3 \sim 1.5 \%$.

\section{Reactions of the Virtual Life}

The VL's reaction to a user's action depends on the general expectation value of all reactions (Table 3 ) and the effect of VL's properties (Table 5).

Table 3. The general expectation value of an anticipated reaction

\begin{tabular}{|c|c|c|c|c|c|c|}
\hline User & Embracing & Strokng & Neglecting & Shouting & Threatering & Hitting \\
\hline Jumping & 9 & 8 & 5 & 3 & 4 & 5 \\
\hline Seducing & 10 & 10 & 6 & 2 & 2 & 1 \\
\hline Sitting & 9 & 9 & 9 & 6 & 4 & 3 \\
\hline Stretching & 7 & 8 & 9 & 6 & 4 & 2 \\
\hline Neglecting & 5 & 7 & 10 & 7 & 8 & 3 \\
\hline Warning & 5 & 6 & 9 & 10 & 9 & 9 \\
\hline Barking & 3 & 5 & 8 & 10 & 10 & 9 \\
\hline Escaping & 2 & 5 & 5 & 8 & 10 & 10 \\
\hline Biting & 1 & 3 & 2 & 9 & 9 & 10 \\
\hline
\end{tabular}

Table 4. The effect that gives VL's property

\begin{tabular}{|c|c|c|c|c|}
\hline $\begin{array}{c}\text { Property } \\
\text { Reaction }\end{array}$ & Docility & Activity & Fatness & $\begin{array}{c}\text { Physical } \\
\text { Strength }\end{array}$ \\
\hline Jumping & 5 & 4 & -3 & 4 \\
\hline Seducing & 7 & 3 & -1 & 5 \\
\hline Sitting & 3 & -2 & 2 & 0 \\
\hline Stretching & 1 & -3 & 3 & 0 \\
\hline Neglecting & -2 & -3 & 2 & 0 \\
\hline Warning & -4 & -1 & 1 & -1 \\
\hline Barking & -6 & 2 & 0 & 3 \\
\hline Escaping & -7 & 4 & -2 & 4 \\
\hline Biting & 8 & 2 & -3 & 5 \\
\hline
\end{tabular}

The values of Table 3,4 are determined by an experiment.

We select one chromosome by the roulette selection to decide VL's reaction and calculate the expectation value that will be selected each reaction about all kinds of reactions by the next formula.

$$
E_{i}=G_{i}+\sum_{a l l j} A_{i j} \times V_{j}
$$

$E_{j}$ : the expectation value that $i$ th reaction will be selected

$G_{i}$ : the general expectation value about $i$ th reaction
$A_{i j}$ : the proportional factor that $j$ th property is $A_{i j}$ reflected in $i$ th property

$V_{j}:$ the $j$ th property value

Then, the VL's reaction is decided by the roulette selection according to the expectation values.

\section{Experimental Results}

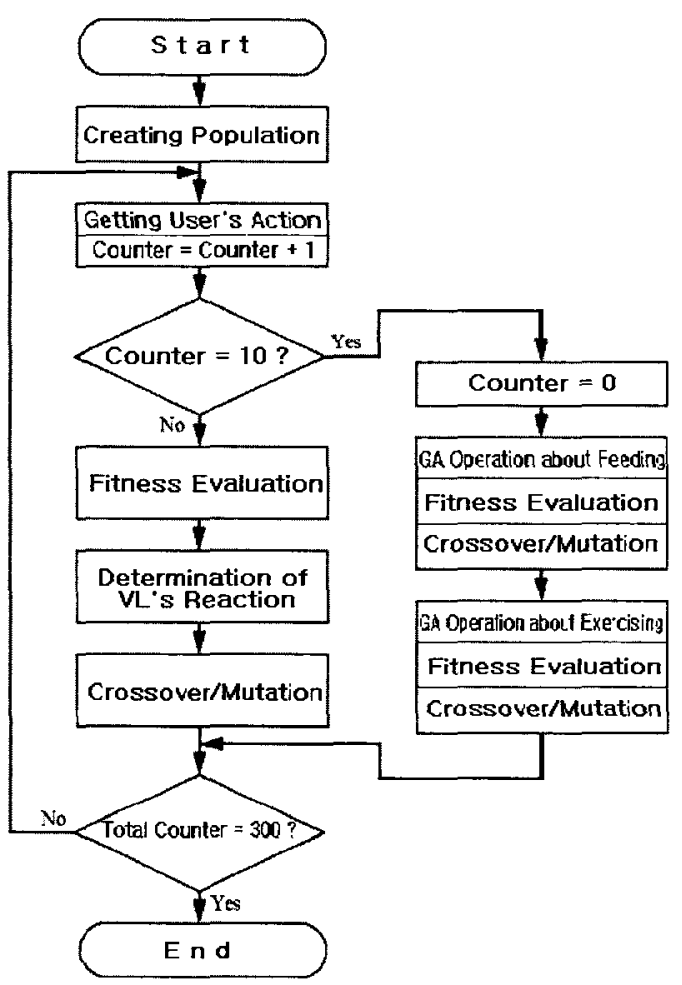

Fig 1. Block diagram of Simulation System

The block diagram of the simulation system is shown in Fig.1.

The input of this simulation system is user's action. In this simulation, it is assumed that the user gives 10 actions in a day and total 300 actions during 30 days to the VL. Every time the user interacts with the VL, the system executes the GA operation, but user's action is feeding or exercising. When a input is feeding or exercising, the system counts the number of such inputs applied in a day, and then the system execute one GA operation for these feeding or exercising inputs at the end of a day.

In the experiments, the user's action is classified into 2 categories (Table 5.). One trains the VL to be gentle and the other does it to be rebellious. In the similar context, the reaction of the $\mathrm{VL}$ is classified into 2 categories (Table 6.). One is a gentle reaction, and the other is a violent reaction. 
퍼지 및 지능시스템 학회 2003, Vol. 13, No. 5

Table 5. Categories of the user's action

\begin{tabular}{|c|c|c|}
\hline Symbol & Action & Category \\
\hline$a$ & embracing & Actions that make the VL docile \\
tendency
\end{tabular}

Table 6. Categories of the VL's reaction

\begin{tabular}{|c|c|c|}
\hline Symbol & Action & Category \\
\hline A & jumping & \multirow{2}{*}{ Reactions of docile tendency } \\
\hline B & seducing & Rean \\
\hline C & sitting & \\
\hline D & stretching & \\
\hline E & neglecting & \multirow{3}{*}{ Reactions of violent tendency } \\
\hline F & warning & \\
\hline$G$ & barking & \multirow{2}{*}{} \\
\hline H & escaping & \\
\hline I & biting & \\
\hline
\end{tabular}

We observe that the VL's properties and the reactions have been changed by the ratio of 2 categories(Table 5) of the user's action during 300 generations. However, the ratio of feeding and exercising tends to be fixed about 2 times and 1 time in a day, since they affect the survival of the VL. Therefore, we use 210 inputs and the associated outputs, to 300 inputs and so on, in order to analyze the simulation results. Next, we present the simulation results for two cases.

\subsection{Simulation I}

In the Simulation I, we assume that people breed a pet in the general manner of the real world. We choose embracing and stroking to be most of the simulation inputs for the user's actions, so that the VL may evolve to be docile. And we choose a few inputs as violent action, in order to observe the change of the VL's reactions and properties, as the user gives some violent action to it, while it grows into a docile one. $70 \%$ of the inputs are the user's actions that make the VL docile and the remaining $30 \%$ are the actions that make the VL violent.

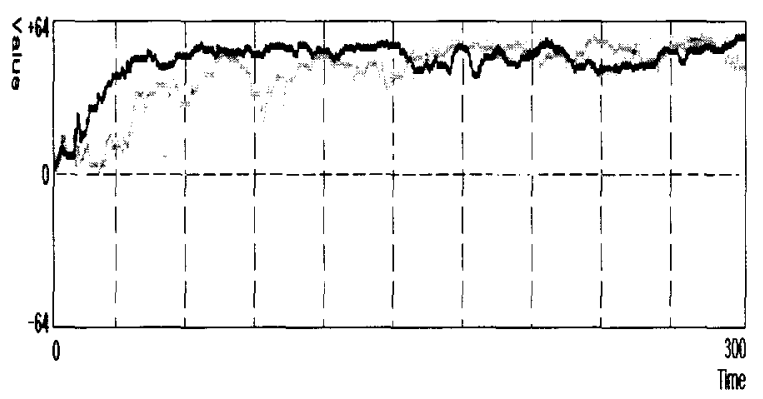

Fig. 2. Change of VL's property mean values on time domain.
In this case, the VL's property values are changed as shown in Fig. 2. The graph shows the change of the mean value of each property value by increasing the user's action.

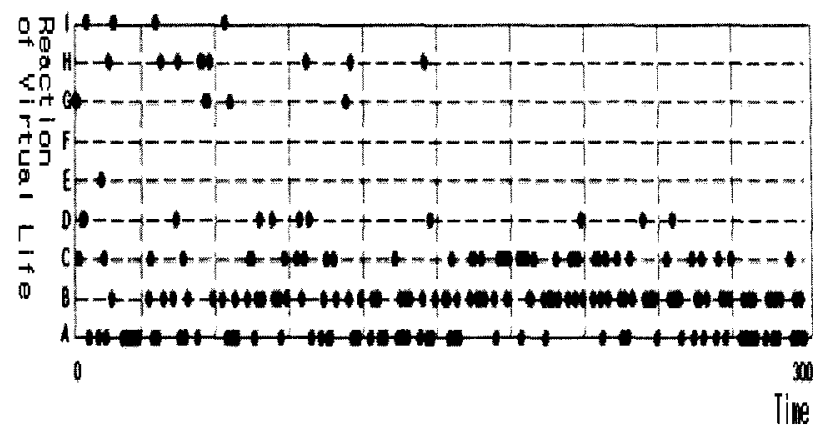

Fig.3. A distribution of VL's reaction in time domain.

The thickest line in Fig. 2 represents the change of the mean value of docility. This line confirms that the VL's behavior converges into docile tendency as the user's actions are on the increase. Fig. 3 displays a distribution of VL's reaction with respect to the increase of user's action.

The VL's reactions are uniformly distributed over ' $A$ ' to ' $\mathrm{I}$ ' before the properties converge to a docile tendency. The VL's reactions are inclined toward a docile tendency after the properties converge to a docile tendency as shown in Fig. 2 3. As a result, we can see that the VL's reaction is influenced by the VL's properties, which converges to a particular tendency. we can confirm this result more definitely in Fig. $4 \sim 5$.

Fig. 4 shows the histograms of the user's action and the VL's reactions for all the users, while Fig. 5 shows the histograms of the 100 repeated experiments under the same conditions.

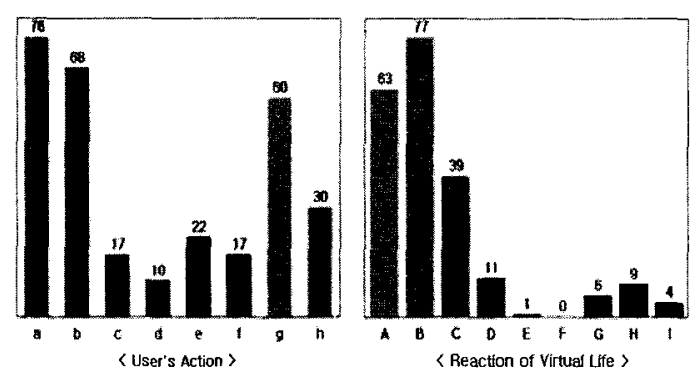

Fig. 4. Histograms of the user's action and the VL's reaction.

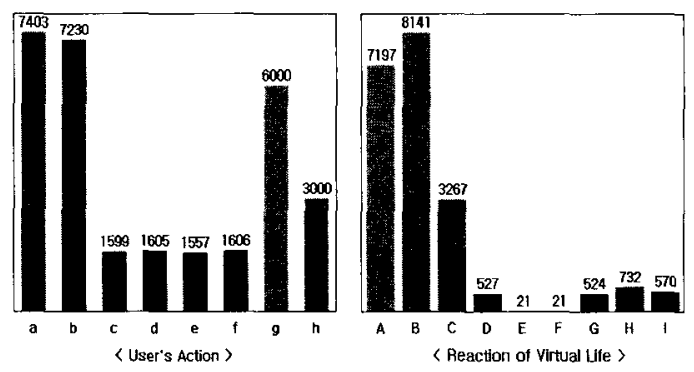

Fig. 5. Histograms of the user's action and the VL's reaction for the 100 repeated experiments. 
Table. 7. Ratios of results in Fig. 5 and Fig. 6

\begin{tabular}{|c|c|c|c|}
\hline \multicolumn{2}{|c|}{} & Fig. 5 & Fig. 6 \\
\hline \multirow{2}{*}{$\begin{array}{c}\text { user's } \\
\text { action }\end{array}$} & to make the VL docile & $68.6 \%$ & $69.7 \%$ \\
\cline { 2 - 4 } & to make the VL violent & $31.4 \%$ & $30.3 \%$ \\
\hline $\begin{array}{c}\text { VL's } \\
\text { reaction }\end{array}$ & a docile tendency & $85.3 \%$ & $88.6 \%$ \\
\cline { 2 - 4 } & a violent tendency & $14.7 \%$ & $11.4 \%$ \\
\hline
\end{tabular}

The ratio of the VL's reaction of violent tendency in the total reaction is less than half of the user's action that makes the VL violent as shown in Table. 7,

\subsection{Simulation II}

In the Simulation $\Pi$, we apply the opposite input pattern in order to compare with the Simulation I. $70 \%$ of the inputs are the user's actions that make the VL violent and the other $30 \%$ of inputs are the actions that make the VL docile. In this case, the VL's property values have changed as in Fig. 6.

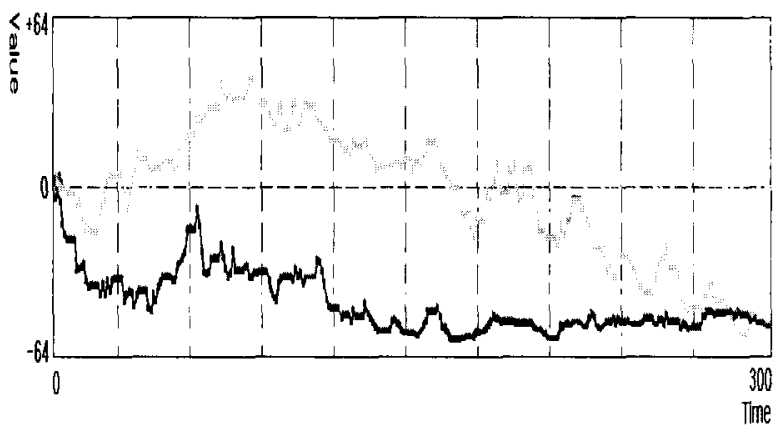

Fig. 6. Change of VL's property mean values on time domain.

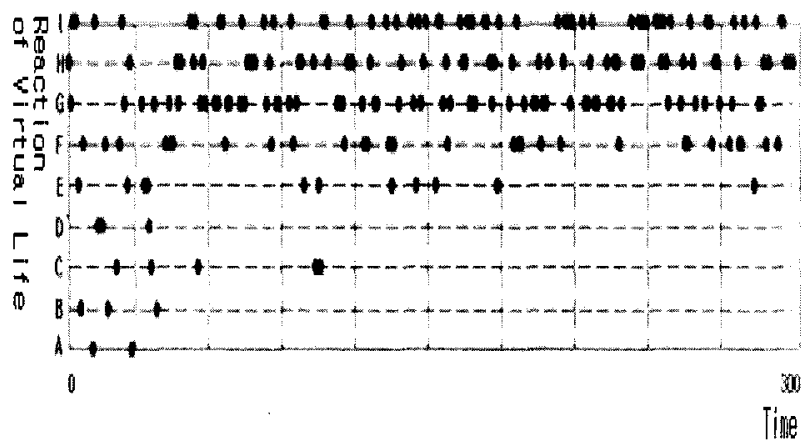

Fig. 7. A distribution of VL's reaction in time domain

The thickest line in Fig. 6 represents the change of the mean value of docility. This line also confirms that the VL converges to a violent tendency as user's actions are on the increase.

The VL's reactions are uniformly distributed over ' $A$ ' to ' $\mathrm{I}$ ' before the properties converge to a violent tendency. The VL's reactions are inclined toward a violent tendency after the properties converge to a violent tendency as shown in Fig. 6 $\sim 7$ and Fig. 8 9.

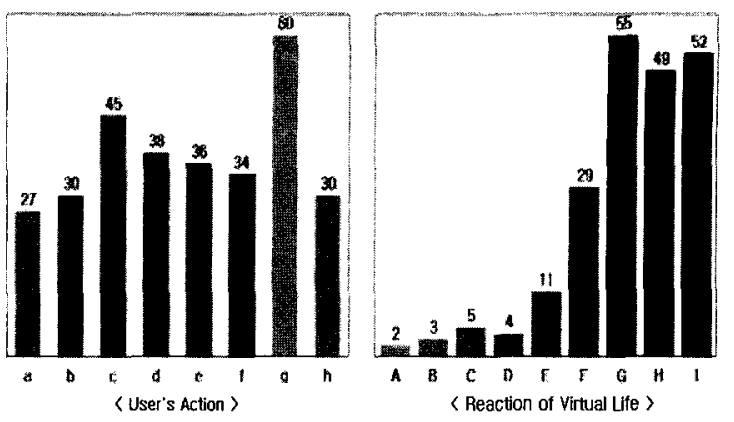

Fig. 8. Histograms of the user's action and the VL's reaction.

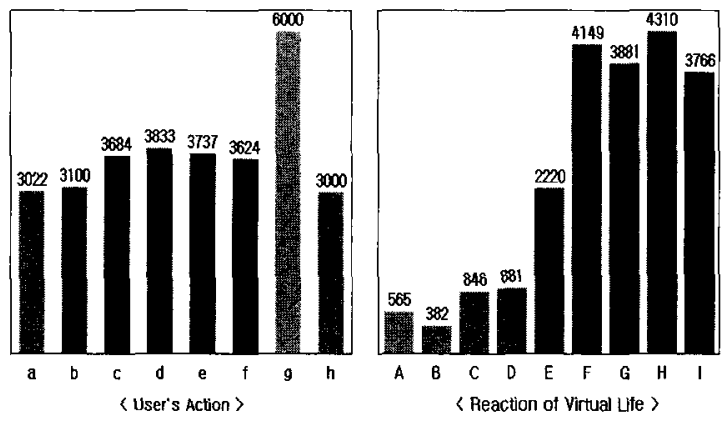

Fig. 9. Histograms of the user's action and the VL's reaction for the 100 repeated experiments.

Table. 8. Ratios of results in Fig. 8 and Fig. 9

\begin{tabular}{|c|c|c|c|}
\hline & Fig. 8 & Fig. 9 \\
\hline \multirow{2}{*}{$\begin{array}{l}\text { user's } \\
\text { action }\end{array}$} & to make the VL docile & $27.1 \%$ & $29.2 \%$ \\
\hline & to make the VL violent & $72.9 \%$ & $70.8 \%$ \\
\hline \multirow{2}{*}{$\begin{array}{c}\text { VL's } \\
\text { reaction }\end{array}$} & a docile tendency & $4.8 \%$ & $8.5 \%$ \\
\hline & a violent tendency & $95.2 \%$ & $91.5 \%$ \\
\hline
\end{tabular}

Similarly, the ratio of the VL's reaction of violent tendency in the total reaction is less than half of the user's action that makes the VL violent in Table. 8,

As demonstrated in the Simulations I II, the growth process of the VL is influenced by the user's action, which results in a higher ratio of the total actions. Moreover, when the VL's properties converge to a particular tendency, they give a dominant affect to the VL's reaction. Finally, when the VL's properties have converged to a particular tendency, they don't change easily as the user gives some actions leading them to another direction. It

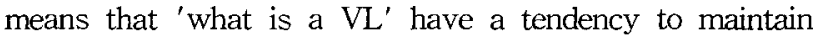
the present status, like the homeostasis.

\section{Conclusion and Future Work}

Important and difficult questions are how to catch the rules of the real life's behaviors and how to model the Virtual Life efficiently on these rules. In our opinion, the most important matters to make the Virtual Life that interacts with the users are the methods to determine the reaction and to change the properties of the Virtual Life. 
In this paper, we modeled, through Genetic Algorithms, the Virtual Life that grows itself and reacts to the user's action according to its own properties. In order to embody this $\mathrm{VL}$ in the online virtual space, much consideration is needed, for example, the interaction with the virtual environment and other VLs.

We are encouraged to find the method that changes dynamically and adaptively some parameters or numerical values that are determined intuitively through experiments.

\section{Reference}

[1] D.E.Goldber, Genetic Algorithms in Search, Optimization, and Machine Leaming, Massachusetts, Addison-Wesley, 1989.

[2] R.L.Haupt and S.E,Haupt, Practical Genetic Algorithms, New York, A Wiley-Interscience Publication, 1998

[3] Banzhaf, W.Interactive Evolution, New York, IOP Publishing Ltd and Oxford Univ. press, 1997.

[4] R.L.Haupt and S.E.Haupt, Practical Genetic Algorithms, New York, Wiley-Interscience, 1999.

[5] Willam M.Spears, Evolutionary Algorithms, Germany, Springer Verlag, 2000.

[6] David B.Fogel, Evolutionary computation, New York, IEEE press, 1999.

[7] Wen Tang and Tao Ruan Wan, "Inteligent Self-Learning Characters for Computer Games", The 20th Eurographics UK Conference (EGUK'02), Leicester, Leicestershire, UK , pp. 51-58, 2002.

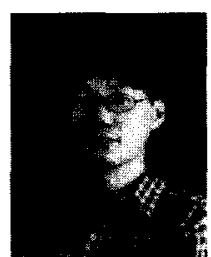

Do-Wan Kim

received the B.S. degree in electrical and electronic engineering from Chung-Ang University, Seoul, Korea, in 2001. He is currently pursuing the M.S. degree. His research interests are Neural Networks and Intelligent Control.

Phone : $+82-2-816-8234$

Fax : $+82-2-816-1856$

E-mail : angeldarker@sirius.cie.cau.ac.kr

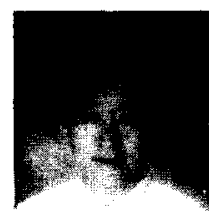

\section{Hoon Kang}

received the B.S. and M.S. degrees in electronic engineering from Seoul National University, Seoul, Korea, in 1982 and 1984, respectively. He received the Ph.D. degree in electrical engineering in Georgia Institute of Technology in 1989. Since 1992, he has been a faculty member of School of Electrical and Electronic Engineering at Chung-Ang University. His research interests are Fuzzy Systems, Artificial Life, Cellular Automata, Neural Networks, Pattern Recognition, Evolutionary Computation, Vision System, Robotics and Intelligent Control.

Phone : $+82-2-820-5320$

Fax : $+82-2-816-1856$

E-mail : hkang@cau.ac.kr

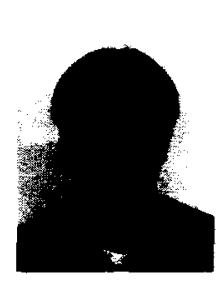

\section{Min-Su Kwon}

received the B.S. degree in electrical and electronic engineering from Chung-Ang University, Seoul, Korea, in 2001. He is currently pursuing the M.S. degree. His research interests are Genetic Algorithms and Atificial Intelligent.

Phone : +82-2-816-8234

Fax : +82-2-816-1856

$\mathrm{E}-$ mail : impactblue@sirius.cie.cau.ac.kr 